\title{
Modeling of Axially Loaded Nanowires Embedded in Elastic Substrate Media with Inclusion of Nonlocal and Surface Effects
}

\author{
Suchart Limkatanyu, ${ }^{1}$ Nattapong Damrongwiriyanupap, ${ }^{2}$ \\ Woraphot Prachasaree, ${ }^{1}$ and Worathep Sae-Long ${ }^{1}$ \\ ${ }^{1}$ Department of Civil Engineering, Faculty of Engineering, Prince of Songkla University, Songkhla 90112, Thailand \\ ${ }^{2}$ Civil Engineering Program, School of Engineering, University of Phayao, Phayao 56000, Thailand
}

Correspondence should be addressed to Suchart Limkatanyu; suchart.1@psu.ac.th

Received 12 July 2013; Accepted 2 August 2013

Academic Editor: Chunyi Zhi

Copyright $\odot 2013$ Suchart Limkatanyu et al. This is an open access article distributed under the Creative Commons Attribution License, which permits unrestricted use, distribution, and reproduction in any medium, provided the original work is properly cited.

\begin{abstract}
Nonlocal and surface effects are incorporated into a bar-elastic substrate element to account for small-scale and size-dependent effects on axial responses of nanowires embedded in elastic substrate media. The virtual displacement principle, employed to consistently derive the governing differential equation as well as the boundary conditions, forms the core of the displacementbased finite element formulation of the nanowire-elastic substrate element. The element displacement shape functions, analytically derived based on homogeneous solution to the governing differential equilibrium equation of the problem, result in the exact element stiffness matrix and equivalent load vector. Two numerical simulations employing the proposed model are performed to study characteristics and behavior of the nanowire-substrate system. The first simulation involves investigation of responses of the wire embedded in elastic substrate. The second examines influences of several system parameters on the contact stiffness and reveals the size-dependent effect on the effective Young's modulus of the system.
\end{abstract}

\section{Introduction}

From early 1990s, science and technology advancement has shifted into the era of nanotechnology, thanks to the discovery of carbon nanotubes by Iijima [1] at NEC Laboratory, Tsukuba, Japan. Many researchers have since then turned their attention to this emerging technology.

Nanostructure has attracted many scientists and engineers due to its superior physical properties and its vast area of novel applications [2]. To broaden its potential and to further understand its mechanical behaviors, experimental and/or theoretical studies at nanoscale are required.

Experimental tests on nanostructures are extremely difficult to be conducted and are oftentimes prohibitively expensive. Numerical simulations have thus been widely used instead in the research community to characterize its mechanical properties and material responses. Generally in nanostructure modeling, there are two categories of numerical simulation approaches: atomistic approach and continuum-mechanics approach.
The atomistic approach, as its name stipulates, focuses on atomic modeling and embraces several techniques, such as classical molecular dynamics [3], tight binding molecular dynamics [4], and density functional theory [5]. Simulation performed using the atomistic approach provides comprehensive details but is still hampered by high computational costs; hence only systems with small numbers of molecule and atom can be practically investigated using this approach [6].

The alternative continuum-mechanics approach serves as another tool option to characterize the nanostructural response and is applicable to large-scale nanostructural system modeling. However, small-scale effect and sizedependent effect inherent to all nanosized structures have yet to be considered and incorporated into the classical continuum-mechanics theory.

Small-scale effect is caused by long-range interatomic interactions while size-dependent effect is a result of energy correspondent to the atoms at the free surface of the nanosized structure. To account for these two effects, two 
nonclassical elasticity theories, nonlocal elasticity theory and surface elasticity theory, have later been incorporated into the classical continuum-mechanics theory.

The nonlocal elasticity theory was initiated by Eringen [7-9] and Eringen and Edelen [10] and has been widely used to study bending, buckling, vibration, and wave propagation of nanostructure [11-18]. The essence of this theory is in its assertion that the stress at a reference point depends on the strain not only at a particular point but also at all other points throughout the elastic body. Interatomic forces and atomic length scale are considered in the material constitutive relation as nonlocal model parameters.

The surface elasticity theory was first proposed by Gurtin and Murdoch $[19,20]$ and has been employed to represent the size-dependent effect caused by the surface stress and the surface elasticity at nanoscale. With the size of a structure approaching a nanoscale regime, the surface free energy caused by the surface stress and surface elasticity could no longer be neglected in comparison with the bulk energy as that in the classical continuum-mechanics theory due to the high surface-to-volume ratio.

Several researchers have incorporated these two surface effects into the classical continuum-mechanics theory to study bending, buckling, vibration, and wave propagation of nanostructure, as afore mentioned [15, 17, 21-24].

Nanowires have become popular with their superior mechanical, electrical, and thermal performance and have drawn considerable interests in nanoscience and nanoengineering. They have been found to fit a wide range of applications covering micro-/nanoelectromechanical systems (M/NEMS), biosensors, optoelectronics, and biotechnology [25-27]. Numerical studies done to date have mainly focused on bending of nanowires. For example, He and Lilley [22] incorporated Gurtin-Murdoch surface model into EulerBernoulli beam model to study surface effect on the bending behavior of nanowires; Jiang and Yan [28] enhanced the nanowire model of $\mathrm{He}$ and Lilley [22] using Timoshenko beam theory to account for the shear deformation; Liu et al. [24] combined the elastica theory with the Gurtin-Murdoch surface model to perform large-displacement analyses of nanowires including the surface effect; Juntarasaid et al. [15] subsequently improved the nonlinear nanowire model of Liu et al. [24] by including the nonlocal effect.

In nanodevices, nanowires are usually integrated into larger structures through substrate media. Therefore, the interaction mechanism between the nanowire and the surrounding substrate media plays an important role in controlling the performance and characterizing the response of those nanodevices. Several researchers have recently investigated the problem of nanowires resting on elastic substrate media. For example, Zhang and Zhao [29] developed a nanowire model lying on an adhesive receding contact foundation; Khajeansari et al. [30] performed parametric studies of silver nanowires resting on Winkler-Pasternak elastic substrate media using an analytical solution to the problem; Malekzadeh and Shojaee [17] investigated surface and nonlocal effects on nonlinear free vibration of nanowires supported by an elastic medium using both Euler-Bernoulli beam theory and Timoshenko beam theory.
In this research, an axially loaded nanowire embedded in an elastic substrate medium is of main interest. Although several numerical models have been developed to perform buckling analyses of nanowire-elastic substrate systems under compression [31-33], to the best knowledge of the authors, no studies under tension have yet to be found. Thus, such a numerical model in this paper is presented for the first time with the idea stemming from axially loaded pile problems [34].

In the study, brief introductions to nonlocal elasticity theory and surface elasticity theory are firstly presented. The two theories are then employed to explain small-scale and size-dependent effects on the response of a nanowire embedded in an elastic substrate medium. The governing differential equilibrium and compatibility equations and constitutive relations of the problem are then presented. Subsequently the Navier-Cauchy governing equation and associated end-boundary force conditions are derived based on the virtual displacement principle and solved analytically to obtain the exact displacement interpolation functions. This notion stems from the Winkler-based beam model developed by Limkatanyu et al. [35]. Formulation of the nanowiresubstrate element is also based on the virtual displacement principle.

Two numerical simulations employing the proposed model are finally performed to study the characteristics and behaviors of the nanowire-substrate system; the first involves investigation of responses of the embedded nanowire and the second examines the influences of several system parameters on contact stiffness and shows the size-dependent effect on the effective Young's modulus of the system.

\section{Small-Scale and Size-Dependent Effects on Nanowires}

Small-scale and size-dependent effects on the response of a nanowire embedded in an elastic substrate medium are incorporated into the proposed model through the following two nonclassical elasticity theories: (i) nonlocal elasticity theory and (ii) surface elasticity theory. Brief descriptions of these two nonclassical elasticity theories are as follows.

2.1. Nonlocal Elasticity Theory. In opposition to classical elasticity theory, nonlocality in the nonlocal elasticity theory implies that the stress field at a generic point $\mathbf{x}$ in an elastic body is not only a function of the strain at a particular point but also a function of strains at other points throughout the body [10]. This nonlocal attribute of the nonlocal elasticity theory conforms well to the atomic theory of lattice dynamics as well as some experimental evidence on phonon dispersion [11].

The nonlocal stress tensor $\boldsymbol{\sigma}^{\mathrm{NL}}$ at a point $\mathbf{x}$ in a nonlocal elastic continuum can be represented as a weighted integral of strain contributions of all material points throughout the body:

$$
\boldsymbol{\sigma}^{\mathrm{NL}}=\int_{V} K\left(\left|\mathbf{x}^{\prime}-\mathbf{x}\right|, \tau\right) \boldsymbol{\sigma}^{L}\left(\mathbf{x}^{\prime}\right) d V\left(\mathbf{x}^{\prime}\right)
$$


where $K\left(\left|\mathbf{x}^{\prime}-\mathbf{x}\right|, \tau\right)$ is the elastic spatial kernel function and represents the nonlocal modulus; $\left|\mathbf{x}^{\prime}-\mathbf{x}\right|$ is the Euclidean distance between material points $\mathbf{x}$ and $\mathbf{x}^{\prime}$; the local classical stress tensor $\sigma^{L}\left(\mathbf{x}^{\prime}\right)$ is the double-dot contraction between the fourth-order elastic tensor $\mathbf{C}\left(\mathbf{x}^{\prime}\right)$ and the strain tensor $\boldsymbol{\varepsilon}\left(\mathbf{x}^{\prime}\right)$; and the dimensionless material constant $\tau$, as defined, is

$$
\tau=\frac{e_{0} a}{L}
$$

where $e_{0}$ is a calibrated material constant in obtaining a good correlation between the model results and analytical or experimental results; $a$ and $L$ represent, respectively, the internal and the external characteristic length. In this work, $L$ represents the length of the nanowire.

The integral form of the nonlocal constitutive relation of (1) makes the solution to the problem difficult. Eringen [8] thus presented the elastic spatial kernel function $K\left(\left|\mathbf{x}^{\prime}-\mathbf{x}\right|, \tau\right)$ in the form of a modified Bessel function and subsequently converted the integral constitutive relation into an equivalent differential constitutive relation; thus

$$
\boldsymbol{\sigma}^{\mathrm{NL}}-\left(e_{0} a\right)^{2} \nabla^{2} \boldsymbol{\sigma}^{\mathrm{NL}}=\boldsymbol{\sigma}^{L}=\mathbf{C}: \boldsymbol{\varepsilon},
$$

where $\nabla^{2}=\partial^{2} / \partial x^{2}+\partial^{2} / \partial y^{2}+\partial^{2} / \partial z^{2}$ is the Laplace differential operator.

Since only the normal stress in the $x$ direction is of interest in this work, (3) can be simplified into a onedimensional form as

$$
\sigma_{x x}^{\mathrm{NL}}-\left(e_{0} a\right)^{2} \frac{d^{2} \sigma_{x x}^{\mathrm{NL}}}{d x^{2}}=E_{x x} \varepsilon_{x x},
$$

where $\sigma_{x x}^{\mathrm{NL}}$ and $\varepsilon_{x x}$ are, respectively, the nonlocal normal stress and normal strain in the $x$ direction and $E_{x x}$ is the Young's modulus.

2.2. Surface Elasticity Theory. To account for surface effect on the nanowire response, the Gurtin-Murdoch continuum model [19] is employed. In this nonclassical continuum model, the nanowire cross-section is considered to be consisted of a solid core and an outer surface shell perfectly bonded to its core, as shown in Figure 1. Following the surface theory proposed by Cammarata [36], the outer surface is considered a mathematically zero thickness layer with a distinct elastic modulus from its core material. Since only normal stress in the $x$ direction is of interest in this work, the residual surface stress $\sigma_{x x}^{s}$ and surface strain $\varepsilon_{x x}^{s}$ along the longitudinal direction are related through the degenerated form of the Shuttleworth's equation [37] as

$$
\sigma_{x x}^{s}=\sigma_{x x}^{s 0}+E_{x x}^{s} \varepsilon_{x x}^{s}
$$

where $E_{x x}^{s}$ is the surface elastic modulus and is usually obtained by atomistic simulations or experimental results and $\sigma_{x x}^{s 0}$ is the residual surface stress and is defined in terms of the surface energy density $\Psi$ as

$$
\sigma_{x x}^{s 0}=\Psi+\left.\frac{\partial \Psi}{\partial \varepsilon_{x x}^{s}}\right|_{\varepsilon_{x x}^{s}=0} .
$$

\section{Governing Equations of Nanowires Embedded in Elastic Substrate Medium}

3.1. Differential Equilibrium Equation: Direct Approach. Figure 2(a) shows a nanowire-elastic substrate system. This system comprises a bar representing a nanowire plus smeared tangential springs to account for the substrate interactive forces. In a direct manner, the governing differential equilibrium equation of the system can be derived as follows.

A differential segment $d x$ taken from the nanowireelastic substrate system is as shown in Figure 2(b). The axial equilibrium of the infinitesimal bar segment $d x$ is written as

$$
\frac{d N(x)}{d x}+p_{x}(x)-D_{s}(x)=0,
$$

where $N(x)$ is the bar-section force $p_{x}(x)$ the longitudinal distributed load and $D_{s}(x)$ the substrate interactive force.

It is noteworthy to remark that this system is internally statically indeterminate and internal forces cannot be determined solely by an equilibrium condition since at any system section there are 2 internal force unknown fields, $N(x)$ and $D_{s}(x)$, while only one equilibrium equation is available.

3.2. Compatibility Equations. The bar-section strain $\varepsilon_{x x}(x)$ and substrate deformation $\Delta_{s}(x)$ define the system sectional deformations, and are, respectively, the conjugate-work pairs of the bar-section force $N(x)$ and substrate interactive force $D_{s}(x)$. These two system sectional deformations are related to the bar axial displacement $u_{x}(x)$ through the following compatibility equations:

$$
\varepsilon_{x x}(x)=\frac{d u_{x}(x)}{d x}, \quad \Delta_{s}(x)=u_{x}(x) .
$$

The bar compatibility condition follows the small strain assumption [38] while the substrate compatibility condition obeys the Winkler-foundation hypothesis [39].

3.3. Sectional Force-Deformation Relations. Imposing the bar-section equilibrium and constitutive relations of (4) and (5) and enforcing the bar section kinematics, the bar-section force $N(x)$ can be expressed in terms of the bar-section strain $\varepsilon_{x x}(x)$ as

$$
N(x)-\left(e_{0} a\right)^{2} \frac{d^{2} N(x)}{d x^{2}}=(E A)^{*} \varepsilon_{x x}(x)+\sigma_{x x}^{s 0} \Gamma,
$$

where $(E A)^{*}=A E_{x x}+\Gamma E_{x x}^{s}$ is the effective bar axial stiffness and accounts for the contributions from the solid core and the outer surface; $A$ is the bar sectional area; and $\Gamma$ is the bar perimeter.

For cross-sections that are circular (Figure 1(b)) and rectangular (Figure $1(\mathrm{c})$ ), the effective bar axial stiffness $(E A)^{*}$ is

$$
\begin{aligned}
(E A)^{*} & =E_{x x} \frac{\pi}{4} D^{2}+E_{x x}^{s} \pi D: \text { circular } \\
& =E_{x x} B H+2 E_{x x}^{s}(B+H) \text { : rectangular, }
\end{aligned}
$$

where $D$ is the diameter in the circular cross section and $B$ and $H$ are, respectively, the width and the height in the rectangular section. 


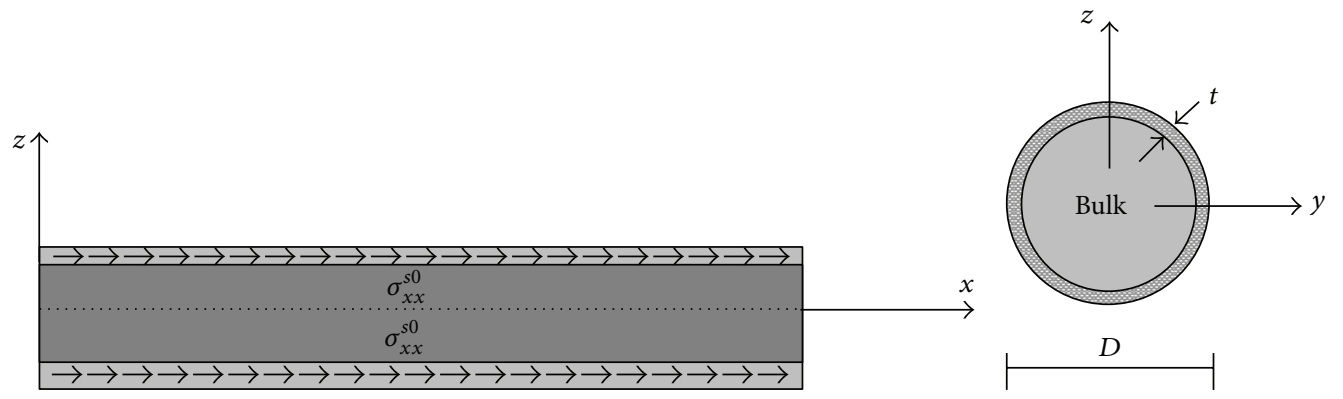

(a)

(b)

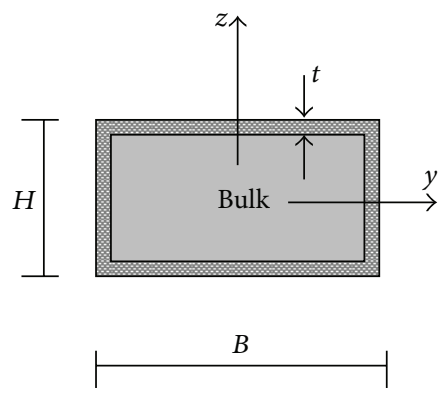

(c)

FIGURE 1: (a) A nanowire with residual surface stress; (b) a circular cross section with a surface layer; (c) a rectangular cross section with a surface layer.

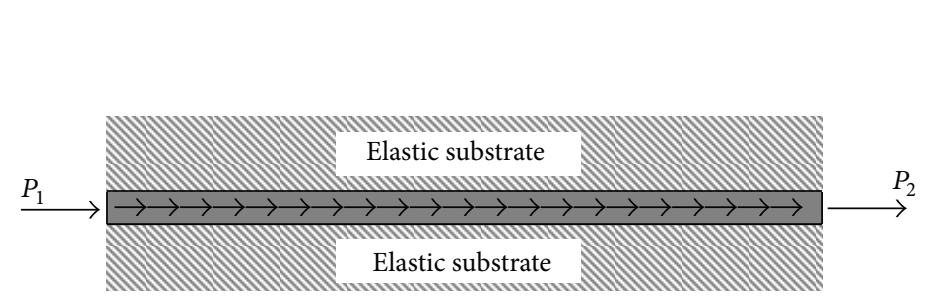

(a)

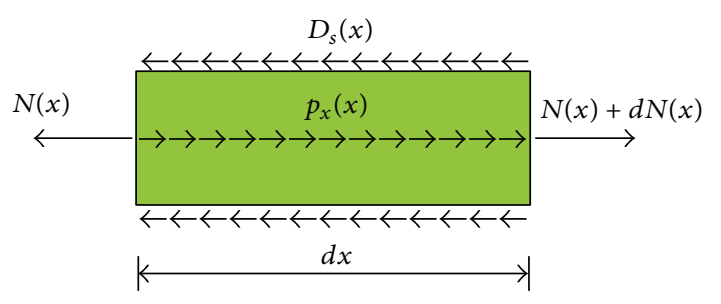

(b)

Figure 2: (a) An axially loaded nanowire-elastic substrate system; (b) a differential segment of an axially loaded nanowire-elastic substrate system.

The substrate interactive force $D_{s}(x)$ is related to the substrate deformation $\Delta_{s}(x)$ as

$$
D_{s}(x)=k_{s} \Delta_{s}(x),
$$

where $k_{s}$ is the elastic substrate stiffness, and is computed as the product of the substrate-stiffness coefficient $K_{s}$ and bar perimeter $\Gamma$.

The equilibrium, compatibility, and constitutive equations for the nanowire embedded in an elastic substrate medium presented above can be conveniently represented in the classical Tonti's diagram of Figure 3.

3.4. The Virtual Displacement Principle: Differential Equilibrium Equation and Natural Boundary Conditions. Virtual displacement equation is an integral expression of the system equilibrium and can be expressed in the general form as

$$
\delta W=\delta W_{\mathrm{int}}+\delta W_{\mathrm{ext}}=0
$$

where $\delta W$ is the system total virtual work; $\delta W_{\text {int }}$ is the system internal virtual work; and $\delta W_{\text {ext }}$ is the system external virtual work.

In the case of a nanowire embedded in an elastic substrate medium, $\delta W_{\text {int }}$ and $\delta W_{\text {ext }}$ can be expressed as

$$
\begin{gathered}
\delta W_{\mathrm{int}}=\int_{L} N(x) \delta \varepsilon_{x x}(x) d x+\int_{L} D_{s}(x) \delta \Delta_{s}(x) d x, \\
\delta W_{\mathrm{ext}}=-\int_{L} p_{x}(x) \delta u_{x}(x) d x-\delta \mathbf{U}^{T} \mathbf{P},
\end{gathered}
$$

where $p_{x}(x)$ is the applied distributed load; the vector $\mathbf{U}=$ $\left\{\begin{array}{ll}U_{1} & U_{2}\end{array}\right\}^{T}$ contains displacements at the bar ends; and the vector $\mathbf{P}=\left\{\begin{array}{ll}P_{1} & P_{2}\end{array}\right\}^{T}$ contains the conjugate-work forces acting at the bar ends. 
Prescribed end displacements

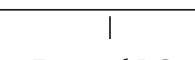

Essential BCs

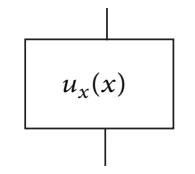

Compatibility I

$\varepsilon_{x x}(x)=\frac{d u_{x}(x)}{d x}$,

$\Delta_{s}(x)=u_{x}(x)$

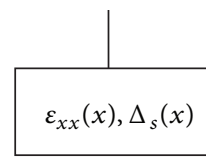

$$
N(x)-\left(e_{0} a\right)^{2} \frac{d^{2} N(x)}{d x^{2}}=(E A)^{*} \varepsilon_{x x}+\sigma_{x x}^{s 0} \Gamma
$$
Material constitutive

FIGURE 3: Tonti's diagram for nanowire embedded in elastic substrate medium: governing differential equations.

Imposing the equilibrium condition of (7), compatibility condition of (8), and constitutive relation of (9) and (11), (12) can be rewritten as

$$
\begin{aligned}
\delta W= & \int_{L}\left((E A)^{*}+k_{s}\left(e_{0} a\right)^{2}\right) \frac{d u_{x}(x)}{d x} \frac{d \delta u_{x}(x)}{d x} d x \\
& -\int_{L}\left(e_{0} a\right)^{2} \frac{d p_{x}(x)}{d x} \frac{d \delta u_{x}(x)}{d x} d x \\
& +\int_{L} \sigma_{x x}^{s 0} \Gamma \frac{d \delta u_{x}(x)}{d x} d x+\int_{L} k_{s} u_{x}(x) \delta u_{x}(x) d x \\
& -\int_{L} p_{x}(x) \delta u_{x}(x) d x-\delta \mathbf{U}^{T} \mathbf{P}=0 .
\end{aligned}
$$

In order to move all differential operators to the axial displacement $u_{x}(x)$, integration by parts is applied once to the first three terms of (14), resulting in the following expression:

$$
\begin{aligned}
\int_{L} \delta u_{x}(x) & {\left[\left((E A)^{*}+k_{s}\left(e_{0} a\right)^{2}\right) \frac{d^{2} u_{x}(x)}{d x^{2}}\right.} \\
& \left.-k_{s} u_{x}(x)+p_{x}(x)-\left(e_{0} a\right)^{2} \frac{d^{2} p_{x}(x)}{d x^{2}}\right] d x
\end{aligned}
$$

Following the Cartesian sign convention, (15) can be written as

$$
\begin{gathered}
\int_{L} \delta u_{x}(x)\left[\left((E A)^{*}+k_{s}\left(e_{0} a\right)^{2}\right) \frac{d^{2} u_{x}(x)}{d x^{2}}\right. \\
\left.-k_{s} u_{x}(x)+p_{x}(x)-\left(e_{0} a\right)^{2} \frac{d^{2} p_{x}(x)}{d x^{2}}\right] d x \\
-\delta U_{1}\left(P_{1}-\left(\left((E A)^{*}+k_{s}\left(e_{0} a\right)^{2}\right) \frac{d u_{x}(x)}{d x}\right.\right. \\
\left.\left.-\left(e_{0} a\right)^{2} \frac{d p_{x}(x)}{d x}+\sigma_{x x}^{s 0} \Gamma\right)_{x=0}\right) \\
-\delta U_{2}\left(P_{2}-\left(\left((E A)^{*}+k_{s}\left(e_{0} a\right)^{2}\right) \frac{d u_{x}(x)}{d x}\right.\right. \\
\left.\left.-\left(e_{0} a\right)^{2} \frac{d p_{x}(x)}{d x}+\sigma_{x x}^{s 0} \Gamma\right)_{x=L}\right)=0 .
\end{gathered}
$$


Due to the arbitrariness of $\delta u_{x}(x)$, the governing differential equilibrium equation of the system described in terms of the axial displacement $u_{x}(x)$ is obtained:

$$
\begin{aligned}
& \left((E A)^{*}+k_{s}\left(e_{0} a\right)^{2}\right) \frac{d^{2} u_{x}(x)}{d x^{2}}-k_{s} u_{x}(x) \\
& \quad=\left(e_{0} a\right)^{2} \frac{d^{2} p_{x}(x)}{d x^{2}}-p_{x}(x), \quad \text { for } x \in(0, L) .
\end{aligned}
$$

Equation (17) is also known as the Navier-Cauchy equation and is used to obtain the analytically derived displacement shape functions. It is noteworthy to mention that for the solution to (17), both nonlocal and surface effects affect the homogeneous part while only nonlocal effect modifies the particular part. When both nonlocal and surface effects are neglected, (17) becomes the governing differential equilibrium equation for a local bar embedded in an elastic medium widely used in the study of axially loaded pile problems.

The natural boundary conditions are obtained by accounting for the arbitrariness of $\delta \mathbf{U}$ in (16) as

$$
\begin{array}{r}
P_{1}=\left(\left((E A)^{*}+k_{s}\left(e_{0} a\right)^{2}\right) \frac{d u_{x}(x)}{d x}\right. \\
\left.-\left(e_{0} a\right)^{2} \frac{d p_{x}(x)}{d x}+\sigma_{x x}^{s 0} \Gamma\right)_{x=0}, \\
P_{2}=\left(\left((E A)^{*}+k_{s}\left(e_{0} a\right)^{2}\right) \frac{d u_{x}(x)}{d x}\right. \\
\left.-\left(e_{0} a\right)^{2} \frac{d p_{x}(x)}{d x}+\sigma_{x x}^{s 0} \Gamma\right)_{x=L} .
\end{array}
$$

Unlike in classical problem of bar-foundation system, (18) indicates that the nonlocal parameter $\left(e_{0} a\right)$ renders the substrate medium stiffness $k_{s}$ as well as the applied distributed load $p_{x}(x)$, presented in the natural boundary conditions. The residual surface stress $\sigma_{x x}^{s 0}$ also modifies the end-force boundaries.

\section{Finite Element Formulation of Nanowires Embedded in Elastic Substrate Media}

4.1. Displacement-Based Formulation. Configuration of the displacement-based nanowire element embedded in elastic substrate medium is shown in Figure 4. In this type of finite element formulation, the axial displacement $u_{x}(x)$ serves as a primary variable and is related to the element nodal displacements $\mathbf{U}$ through the displacement shape function matrix $\mathbf{N}_{B}(x)$ :

$$
u_{x}(x)=\mathbf{N}_{B}(x) \mathbf{U}
$$

The displacement shape functions in $\mathbf{N}_{B}(x)$ are obtained by solving analytically the governing differential equilibrium equation of (17), as will be discussed in the subsequent section. The bar and elastic substrate compatibility conditions are both enforced in the strong sense. The bar-section strain $\varepsilon_{x x}(x)$ and substrate deformation $\Delta_{s}(x)$ are related to

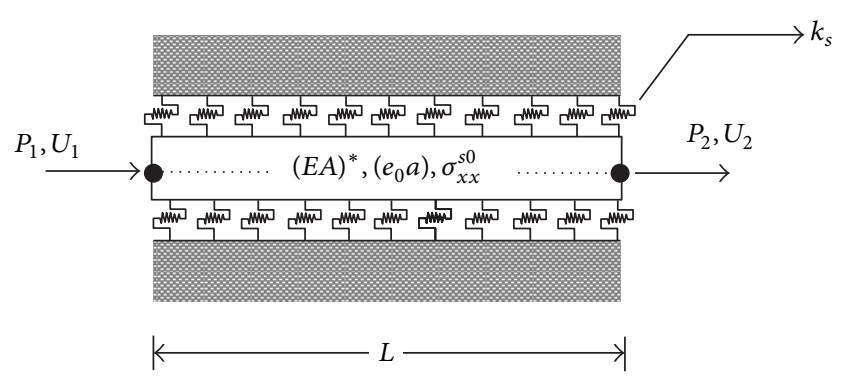

FIGURE 4: Nanowire-substrate element.

the element nodal displacements $\mathbf{U}$ through the following equations:

$$
\begin{gathered}
\varepsilon_{x x}(x)=\frac{d \mathbf{N}_{B}(x)}{d x} \mathbf{U}=\mathbf{B}_{B}(x) \mathbf{U}, \\
\Delta_{s}(x)=\mathbf{N}_{B}(x) \mathbf{U}=\mathbf{B}_{s}(x) \mathbf{U},
\end{gathered}
$$

where $\mathbf{B}_{B}(x)$ and $\mathbf{B}_{s}(x)$ are, respectively, the bar and substrate deformation-displacement matrices.

An alternative way to express the system equilibrium equation is that the virtual displacement principle forms the core of the formulation in the displacement-based model. Applying the virtual displacement principle of (12), substituting the compatibility conditions of (20), imposing the constitutive relation of (9) and (11), and subsequently imposing the arbitrariness of the virtual nodal displacements $\delta \mathbf{U}$ result in the following element stiffness equation:

$$
\left(\mathbf{K}_{B B}+\mathbf{K}_{s s}\right) \mathbf{U}=\mathbf{P}+\mathbf{P}^{p_{x}}+\mathbf{P}^{\mathrm{NL}}-\mathbf{P}^{s 0}
$$

where $\mathbf{K}_{B B}=\int_{L}\left((E A)^{*}+k_{s}\left(e_{0} a\right)^{2}\right) \mathbf{B}_{B}^{T} \mathbf{B}_{B} d x$ is the bar element stiffness matrix, $\mathbf{K}_{s s}=\int_{L} k_{s} \mathbf{B}_{s}^{T} \mathbf{B}_{s} d x$ is the substrate element stiffness matrix, $\mathbf{P}^{p_{x}}=\int_{L} \mathbf{N}_{B}^{T} p_{x} d x$ is the equivalent load vector due to the distributed load $p_{x}(x), \mathbf{P}^{\mathrm{NL}}=\int_{L}\left(e_{0} a\right)^{2}\left(d p_{x}(x) /\right.$ $d x) \mathbf{B}_{B}^{T} d x$ is the equivalent load vector due to the nonlocal effect, and $\mathbf{P}^{s 0}=\int_{L} \sigma_{x x}^{s 0} \Gamma \mathbf{B}_{B}^{T} d x$ is the equivalent load vector due to the residual surface stress $\sigma_{x x}^{s 0}$.

Analytical expressions of these element matrices and equivalent load vectors are given in Appendices A and B, respectively.

4.2. "Exact" Displacement Shape Functions. In this study, the analytical solution to the governing differential equation of (17) is central to obtain the exact displacement shape functions, resulting in the exact element stiffness matrix and equivalent load vector. The homogeneous solution of (17) for $p_{x}(x)=0$ is given [40] as

$$
u_{x}(x)=\gamma_{1} c_{1}+\gamma_{2} c_{2}
$$

where $c_{1}$ and $c_{2}$ are generalized coordinates to be determined from the geometric boundary conditions and $\gamma_{1}$ and $\gamma_{2}$ are exponential functions, having 


$$
\gamma_{1}=e^{-\lambda x}, \quad \gamma_{2}=e^{\lambda x},
$$

where $\lambda=\sqrt{k_{s} /\left((E A)^{*}+k_{s}\left(e_{0} a\right)^{2}\right)}$.

In matrix form, $(22)$ can be written as

$$
u_{x}(x)=\Gamma^{T} \mathbf{C},
$$

where $\boldsymbol{\Gamma}$ and $\mathbf{C}$ are column vectors containing $\gamma_{1}$ and $\gamma_{2}$ and $c_{1}$ and $c_{2}$, respectively.

The two geometric boundary conditions are related to element nodal displacements as

$$
\left.u_{x}\right|_{x=0}=U_{1},\left.\quad u_{x}\right|_{x=L}=U_{2} .
$$

Substituting $u_{x}(x)$ from (24) into (25) yields the following algebraic relation:

$$
\mathbf{U}=\mathbf{T C}
$$

where $\mathbf{T}$ is the mapping matrix between the generalized coordinates and the element nodal displacements. Symbolically solving (26) and subsequently substituting back into (24), we have

$$
u_{x}(x)=\Gamma^{T} \mathbf{T}^{-1} \mathbf{U}=\mathbf{N}_{B}(x) \mathbf{U},
$$

where $\mathbf{N}_{B}(x)=\left\lfloor N_{B 1}(x) \quad N_{B 2}(x)\right\rfloor$ is an array containing the exact displacement shape functions. The expression of each displacement shape function is given in Appendix C.

All symbolic calculations throughout this paper are performed using the computer software Mathematica [41].

\section{Results and Discussions}

Two numerical simulations employing the proposed model are performed to study the characteristics and behaviors of a nanowire-substrate system. The first simulation involves investigation of the response of the nanowire embedded in an elastic substrate. The second simulation examines the influences of several system parameters on contact stiffness and shows the size-dependent effect on the effective Young's modulus of the system.

5.1. Simulation I: Response of a Nanowire Embedded in an Elastic Substrate. A circular silver nanowire embedded in a substrate medium is subjected to a tensile force $P$ of $2400 \mathrm{nN}$ at its right end as shown in Figure 5. Geometric properties of the silver nanowire follow those used by Juntarasaid et al. [15] and are taken as $L=1000 \mathrm{~nm}$ and $D=50 \mathrm{~nm}$. Material and surface properties of the silver nanowire come from those used by $\mathrm{He}$ and Lilley [22]. The bulk modulus $E_{x x}$ of the silver nanowire is $76 \mathrm{GPa}$ while its residual surface stress $\sigma_{x x}^{s 0}$ and elastic surface modulus $E_{x x}^{\mathcal{S}}$ are 0.89 and $1.22 \mathrm{nN} / \mathrm{nm}$, respectively. These surface properties correspond to the crystallographic direction of [001] as reported by Shenoy [42]. The nonlocal scale parameter, taken as $\left(e_{0} a\right)=200 \mathrm{~nm}$, corresponds to the one used by Juntarasaid et al. [15] and Yang and Lim [16]. For the substrate medium, a stiffness coefficient $K_{s}$ of $95 \times 10^{-3} \mathrm{nN} / \mathrm{nm}^{3}$ is assumed, resulting in
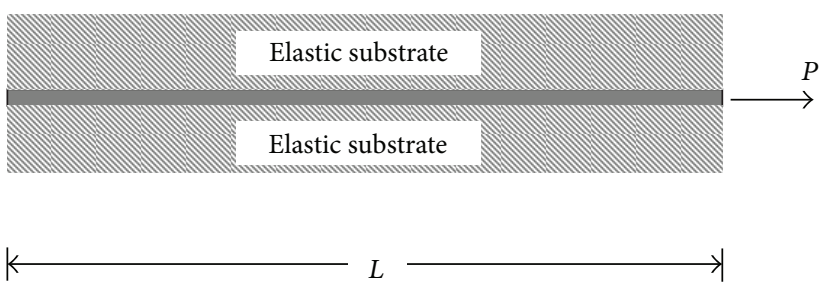

$P=2400 \mathrm{nN} ; L=1000 \mathrm{~nm} ; D=50 \mathrm{~nm} ; E_{x x}=76 \mathrm{GPa} ;$

$E_{x x}^{s}=1.22 \mathrm{nN} / \mathrm{nm} ; \sigma_{x x}^{s 0}=0.89 \mathrm{nN} / \mathrm{nm} ;\left(e_{0} a\right)=200 \mathrm{~nm} ;$

$k_{s}=14.92 \mathrm{nN} / \mathrm{nm}^{2}$

FIgURE 5: Schematic and values used in numerical simulation I.

an elastic substrate stiffness $k_{s}$ of $14.92 \mathrm{nN} / \mathrm{nm}^{2}$. These values for the substrate are meant to represent the polymer as the surrounding substrate medium [43]. Four analytical cases are performed in this example. In the first two cases, surface effect on the nonlocal nanowire-substrate responses is investigated while in the two remaining cases, surface effect on the local nanowire-substrate responses is studied.

Figure 6 compares axial-displacement distributions along the nanowire obtained from the four cases. It can be observed that for these particular values of model parameters, influences of the nonlocal effect are more pronounced than those of the surface effect. The system becomes drastically softer when the nonlocality is neglected. It is interesting to point out that the elastic substrate stiffness is nullified with the disappearance of the nonlocal scale parameter as shown in (17), thus partially resulting in a weaker nanowire-substrate system. A larger portion of the nanowire is mobilized when the nonlocal effect is included. The axial-displacement distribution obtained from the nonlocal model with surface effect can hardly be distinguished from that obtained from the nonlocal model without surface effect. However, the surface effect is slightly magnified when the nonlocal effect is excluded. The most distinctive feature of the surface effect on the axial-displacement response is the nonzero slope of the axial-displacement distribution curve at the free end. This nonzero slope is induced by the residual surface stress and will be discussed later.

Figures 7(a) and 7(b) show the nanowire axial-strain and axial-force distributions obtained from the four analysis cases. Similar to the observation on Figure 6, the influences of the nonlocal effect are more noticeable than those of the surface effect. It is interesting to observe that in Figure 7(a), the nonzero axial strain is noticed at the free end when the surface effect is included. This nonzero axial strain corresponds to the nonzero slope of the axial-displacement distribution at the free end and is caused by the nonzero residual surface stress. In Figure 7(b), the axial-force distribution obtained with the nonlocal model tends to be spreading out along the larger portion of the nanowire than that obtained with the local model. Unlike the axial-displacement and axial-strain distributions, the surface effect on the axial-force distribution is clearly observed, particularly for the nonlocal model.

Figure 8 shows the substrate interactive-force diagrams obtained from the four cases. As expected, the shapes of 


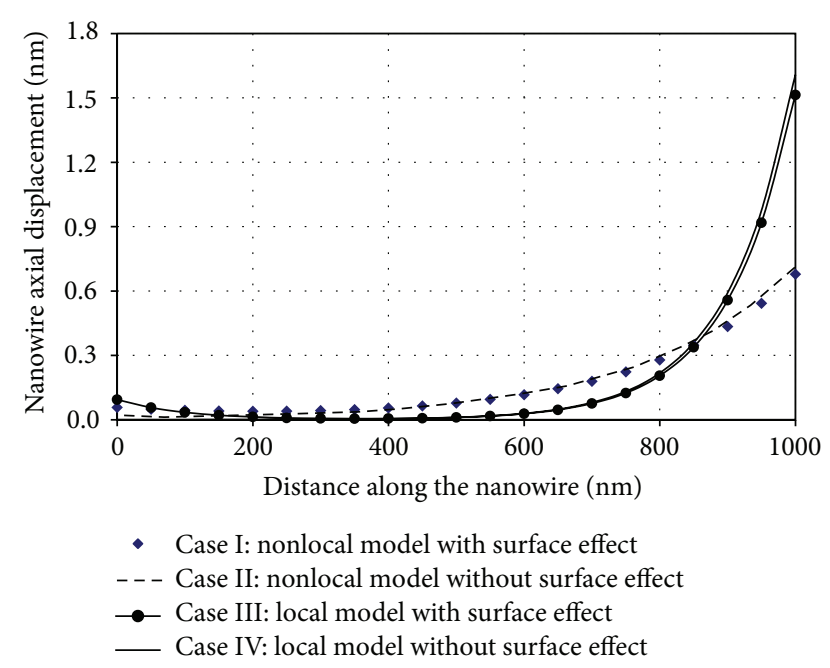

FIGURE 6: Nanowire axial displacement versus distance along the wire.

the substrate interactive-force distributions resemble those of the axial-displacement distributions following the Winklerfoundation model. Similar to the observation made earlier, the nonlocality plays a crucial role in dictating the substrate interactive-force diagram for these particular values of model parameters. The essence of the surface effect can be slightly observed when the nonlocal effect is excluded

\subsection{Simulation II: Parametric Studies of Nanowire-Substrate} Systems. Parametric studies of the nanowire-substrate system in Figure 5 are performed to illustrate the size-dependent effect on the effective Young's modulus of the system and to investigate sensitivity of the model parameters on contact stiffness. As earlier described in Section 5.1., the residual surface stress $\sigma_{x x}^{s 0}$ and elastic surface modulus $E_{x x}^{s}$ are 0.89 and $1.22 \mathrm{nN} / \mathrm{nm}$, respectively. Model parameters investigated herein include the nanowire diameter, the nonlocal parameter, and the substrate stiffness. The slenderness ratio $L / D$ is used to define the nanowire-diameter parameter and varies from 10 to 120 . Therefore, the corresponding nanowire diameter varies from 100 to $8.3 \mathrm{~nm}$. The nonlocal parameter $\left(e_{0} a\right)$ ranges from zero to $100 \mathrm{~nm}$. The following nondimensional parameter $\bar{K}_{s}$ varying from 1 to 10 is used to define the substrate-stiffness parameter:

$$
\bar{K}_{s}=\frac{K_{s} \Gamma L^{2}}{E_{x x} A} .
$$

The size-dependent effect on the effective Young's modulus of the system is now under investigation. Employing a similar approach used by $\mathrm{He}$ and Lilley [22] and Jiang and Yan [28], the effective Young's modulus $E_{B}^{\text {eff }}$ is obtained in the following manner The end displacement $u_{\text {end }}$ of the nanowire-substrate system is first computed using the proposed model, and then $E_{B}^{\mathrm{eff}}$ is obtained by solving the following nonlinear equation:

$$
u_{\mathrm{end}}=\frac{P \operatorname{coth}\left(\sqrt{k_{s} /\left(E_{B}^{\mathrm{eff}} A\right) L}\right)}{\sqrt{k_{s} E_{B}^{\mathrm{eff}} A}},
$$

where the right-hand side of (29) is the end displacement obtained from the proposed model without the nonlocal and surface effects.

Figure 9 shows variation of the effective Young's modulus $E_{B}^{\mathrm{eff}}$ with the diameter $D$ and nonlocal scale parameter $\left(e_{0} a\right)$ for nanowires embedded in substrate media with different substrate-stiffness parameters $\bar{K}_{s}$. In general, effective Young's modulus increases with decreasing nanowire diameter and increasing nonlocal scale parameter, especially for higher value of substrate-stiffness parameters. Thus, nonlocality and nanowire-substrate interactions are deemed essential to characterize the size-dependent effect on the effective Young's modulus of the system. It is also observed that variation of the effective Young's modulus $E_{B}^{\mathrm{eff}}$ with diameter $D$ is not dependent on the substrate-stiffness parameter $\bar{K}_{s}$ when nonlocality is neglected. This is due to the nullification of the substrate stiffness induced by the disappearance of the nonlocal scale parameter as indicated in (17).

Sensitivity analysis of model parameters on the contact stiffness is performed to measure essence of the nonlocal and surface effects on the system response. Following the definition by Jiang and Yan [28] and Khajeansari et al. [30], the contact stiffness of an axially loaded nanowire-substrate system is simply defined as

$$
K^{\mathrm{end}}=\frac{P_{\text {end }}}{u_{\text {end }}}
$$

where $P_{\text {end }}$ and $u_{\text {end }}$ are the imposed force and induced displacement at an end point, respectively.

In this study, two types of normalized contact stiffness are defined and used to assess the essence of the nonlocal and surface effects on the system contact stiffness. The first normalized contact stiffness is used to represent the attribution of the nonlocal effect and is defined as

$$
\bar{K}_{\text {non }}=\frac{K_{n s}^{\text {end }}}{K_{s}^{\text {end }}},
$$

where $K_{n s}^{\text {end }}$ is the contact stiffness accounting for both the nonlocal and surface effect; and $K_{s}^{\text {end }}$ is the contact stiffness accounting for only the surface effect. The second normalized contact stiffness is employed to represent the ascription of the surface effect and is defined as:

$$
\bar{K}_{\text {sur }}=\frac{K_{n s}^{\text {end }}}{K_{n}^{\text {end }}},
$$

where $K_{s}^{\text {end }}$ is the contact stiffness accounting for only the nonlocal effect.

Figure 10 shows influences of the nanowire diameter $D$ and nonlocal scale parameter $\left(e_{0} a\right)$ on the normalized 


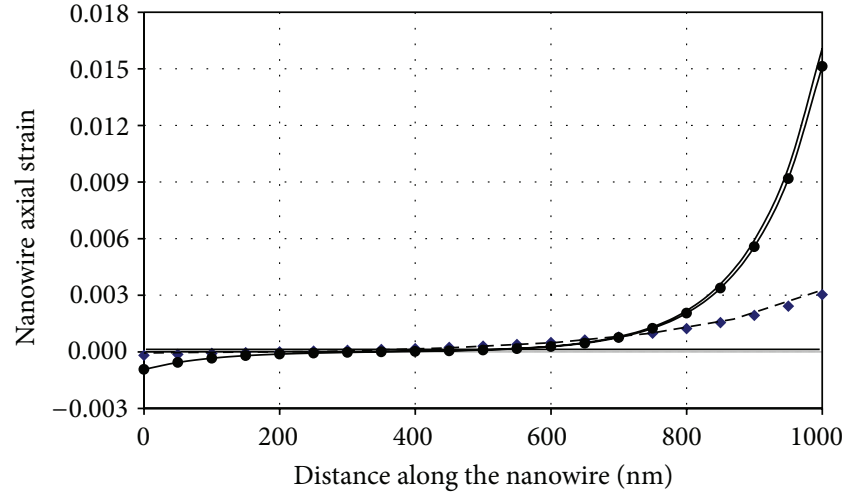

- Case I: nonlocal model with surface effect

- - - Case II: nonlocal model without surface effect

$\rightarrow$ Case III: local model with surface effect

- Case IV: local model without surface effect

(a)

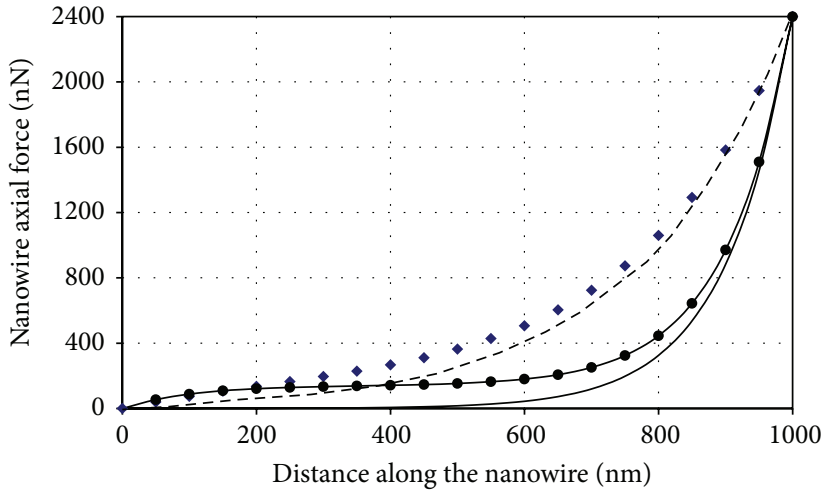

- Case I: nonlocal model with surface effect

- - - Case II: nonlocal model without surface effect

$\rightarrow$ Case III: local model with surface effect

— Case IV: local model without surface effect

(b)

FIgURE 7: Nanowire axial strain (a) and nanowire axial force (b) versus distance along the wire.

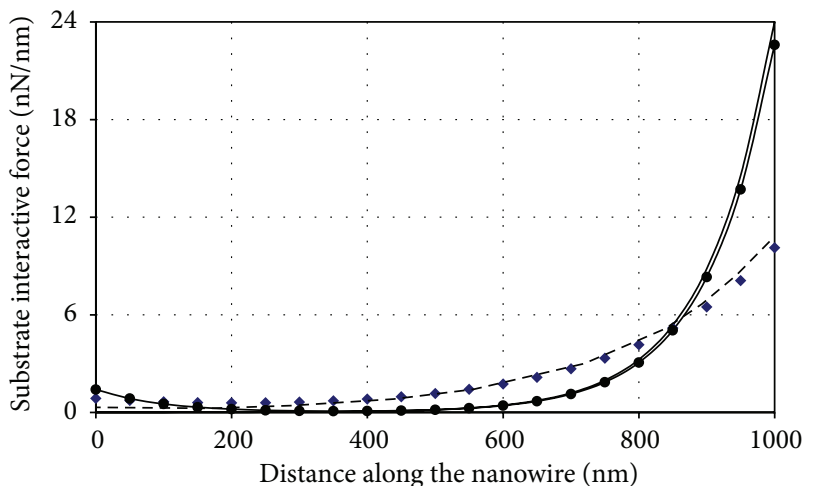

- Case I: nonlocal model with surface effect

- - - Case II: nonlocal model without surface effect

$\rightarrow$ Case III: local model with surface effect

_ Case IV: local model without surface effect

FIGURE 8: Substrate interactive force versus distance along the wire.

nonlocal contact stiffness $\bar{K}_{\text {non }}$ for nanowires embedded in substrate media with different substrate-stiffness parameter $\bar{K}_{s}$. As anticipated, the normalized nonlocal contact stiffness $\bar{K}_{\text {non }}$ is invariant with the nanowire diameter $D$ when the nonlocal effect is neglected $\left(\left(e_{0} a\right)=0 \mathrm{~nm}\right)$. With a larger value of nonlocal scale parameter $\left(e_{0} a\right)$, the nanowire diameter $D$ shows a more significant effect on the normalized nonlocal contact stiffness $\bar{K}_{\text {non }}$, especially for higher values of substrate-stiffness parameters. With increasing slenderness ratio $(L / D)$, the nonlocal effect becomes more pronounced, especially for higher values of substrate-stiffness parameter $\bar{K}_{s}$ and nonlocal scale parameter $\left(e_{0} a\right)$.

Figure 11 shows influences of the nanowire diameter $D$ and the nonlocal scale parameter $\left(e_{0} a\right)$ on the normalized surface contact stiffness $\bar{K}_{\text {sur }}$ for nanowires embedded in substrate media with different substrate-stiffness parameter $\bar{K}_{s}$. It is interesting to observe that the normalized surface contact stiffness $\bar{K}_{\text {sur }}$ is independent of $\bar{K}_{s}$ when nonlocality is not considered $\left(\left(e_{0} a\right)=0 \mathrm{~nm}\right)$. This becomes clear with the consideration of (17) where the elastic substrate stiffness $k_{s}$ is nullified with the disappearance of the nonlocal scale parameter $\left(e_{0} a\right)$. With increasing slenderness ratio $(L / D)$, the surface effect becomes more pronounced, especially for the lower values of substrate-stiffness parameter $\bar{K}_{s}$ and nonlocal scale parameter $\left(e_{0} a\right)$. This observation is in opposition to the one drawn from Figure 10.

Based on the observations made from Figures 10 and 11 , it can be concluded that nonlocal effect is relatively more influential than surface effect for the range of model parameters investigated herein.

\section{Summary and Conclusions}

In this paper, the "exact" element stiffness matrix and the equivalent load vector for an axially loaded nanowiresubstrate system including both nonlocal and surface effects are presented. The exact element stiffness matrix is derived based on the matrix virtual displacement principle using the "exact" displacement shape functions. The exact displacement shape functions are obtained by solving analytically the governing differential equilibrium equation of the problem. Two numerical simulations are performed to study characteristics and behaviors of the nanowire-substrate system.

The first simulation indicates that both nonlocal and surface effects lead to a stiffer nanowire-substrate system in the same way as increasing the nanowire axial rigidity and affect the distributions of local responses along the nanowire.

The second simulation points out that both nonlocal scale parameter and surrounding substrate stiffness magnify the size-dependent effect on the effective Young's modulus of the system. Furthermore, the parametric studies performed in 

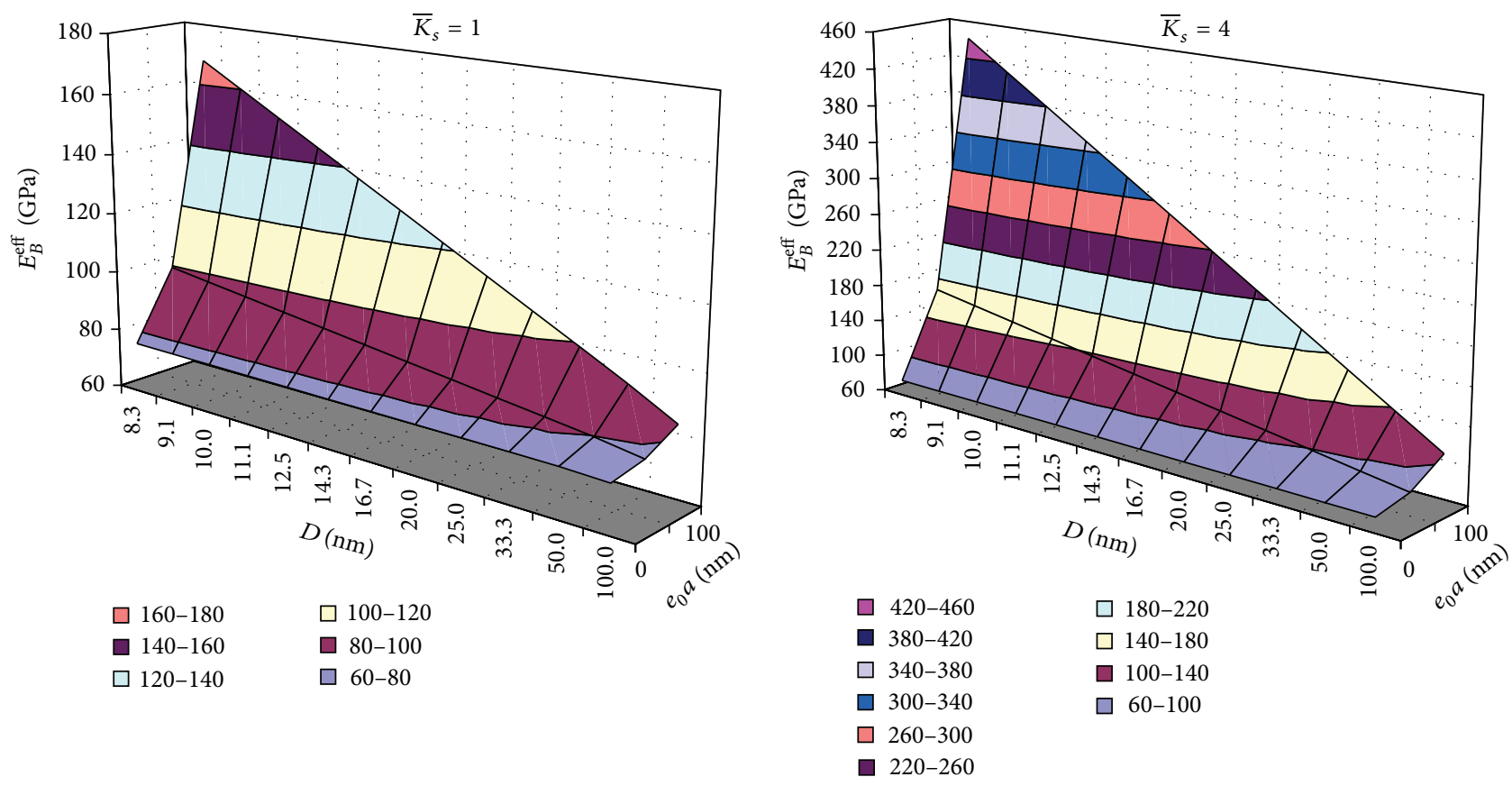

(a)
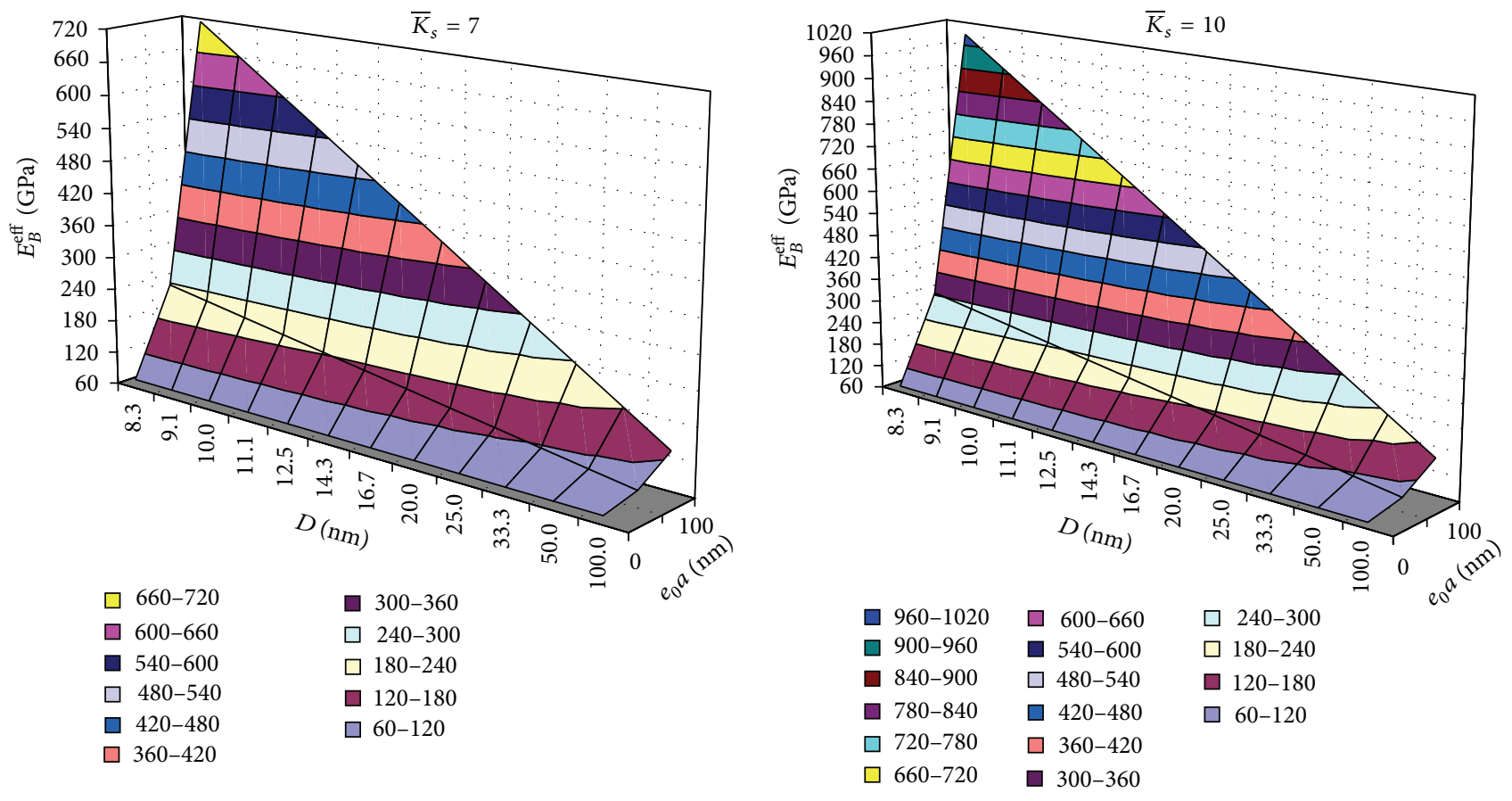

(c)

(d)

FIGURE 9: Variation of effective young's modulus with nanowire diameter and nonlocal scale parameter for various elastic substrate stiffness.

this second numerical simulation show that nonlocal effect is relatively more influential than surface effect for the range of model parameters investigated herein and that the influence of elastic substrate stiffness is nullified with the disappearance of the nonlocal scale parameter.
One next step in this research direction is to include nonlinearities into both the nanowire and the substrate medium. It is hoped that the nanowire-substrate medium element developed herein will be useful to scientists and engineers working in the area of nanoscience and nanoengineering. 


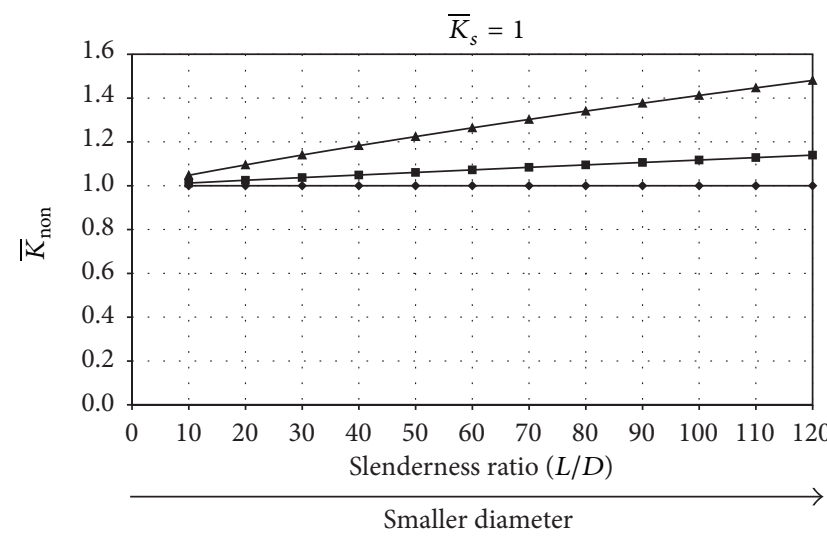

(a)

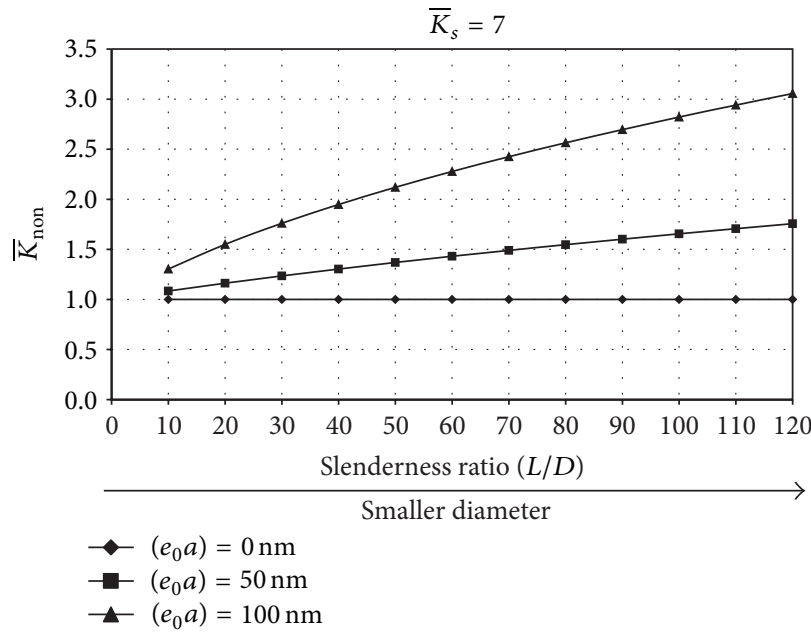

(c)

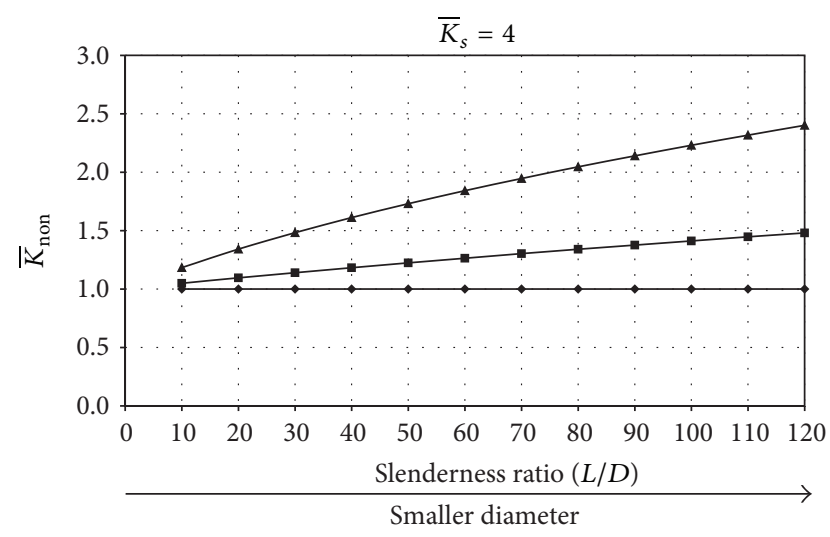

(b)

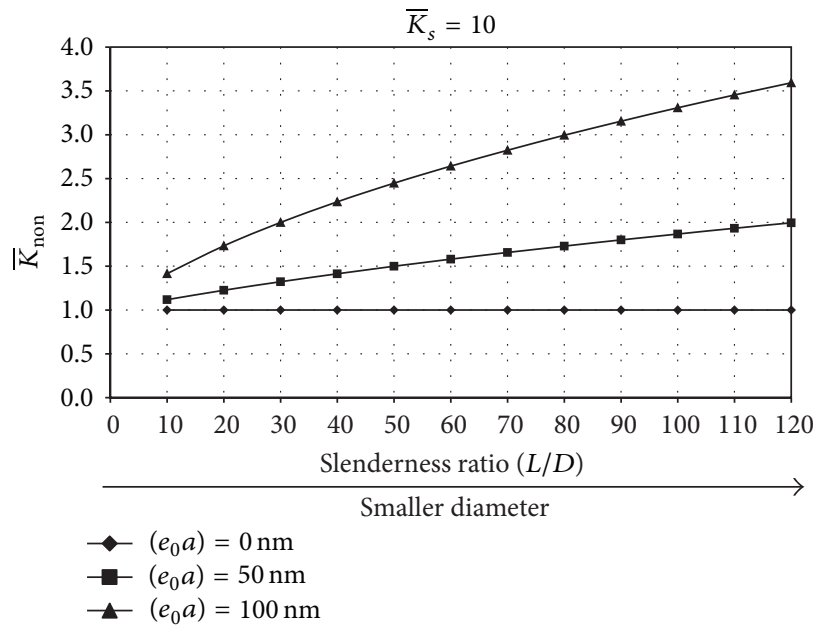

(d)

FIGURE 10: Variation of normalized stiffness $\bar{K}_{\text {non }}$ with $L / D$ for various elastic substrate stiffness and nonlocal scale parameter.

\section{Appendices}

\section{A. Element Stiffness Matrix}

The bar element stiffness matrix may be written as

$$
\mathbf{K}_{B B}=\left[\begin{array}{cc}
K_{11}^{B B} & K_{12}^{B B} \\
\text { Symm. } & K_{22}^{B B}
\end{array}\right] \text {, }
$$

where

$$
\begin{aligned}
& K_{11}^{B B}=K_{22}^{B B}=\frac{k_{s}}{4 \sinh ^{2}[\lambda L]}\left(2 L+\frac{\sinh [2 \lambda L]}{\lambda}\right), \\
& K_{12}^{B B}=K_{21}^{B B}=-\frac{k_{s}}{2 \sinh [\lambda L]}\left(\frac{1}{\lambda}+\frac{L}{\tanh [\lambda L]}\right) .
\end{aligned}
$$

The substrate element stiffness matrix may be written as

$$
\mathbf{K}_{s s}=\left[\begin{array}{cc}
K_{11}^{s s} & K_{12}^{s s} \\
\text { Symm. } & K_{22}^{s s}
\end{array}\right] \text {, }
$$

where

$$
\begin{gathered}
K_{11}^{s s}=K_{22}^{s s}=\frac{k_{s}}{4 \sinh ^{2}[\lambda L]}\left(-2 L+\frac{\sinh [2 \lambda L]}{\lambda}\right), \\
K_{12}^{s s}=K_{21}^{s s}=-\frac{k_{s}}{2 \sinh [\lambda L]}\left(\frac{1}{\lambda}-\frac{L}{\tanh [\lambda L]}\right) .
\end{gathered}
$$

\section{B. Equivalent Element Load Vector}

The equivalent load vector due to a linearly distributed load $p_{y}(x)=p_{0}+\left(\left(p_{L}-p_{0}\right) / L\right) x$ may be written as

$$
\mathbf{P}^{p_{x}}=\left\{\begin{array}{ll}
P_{1}^{p_{x}} & P_{2}^{p_{x}}
\end{array}\right\}^{T}
$$

where

$$
\begin{aligned}
& P_{1}^{p_{x}}=\frac{\left(p_{L}-p_{0}\right)}{\lambda^{2} L}+\frac{1}{\lambda}\left(\frac{\left(p_{0}-p_{L}\right)}{\sinh [\lambda L]}+p_{0} \tanh \left[\frac{\lambda L}{2}\right]\right), \\
& P_{2}^{p_{x}}=\frac{\left(p_{0}-p_{L}\right)}{\lambda^{2} L}+\frac{1}{\lambda}\left(\frac{\left(p_{L}-p_{0}\right)}{\tanh [\lambda L]}+p_{0} \tanh \left[\frac{\lambda L}{2}\right]\right) .
\end{aligned}
$$




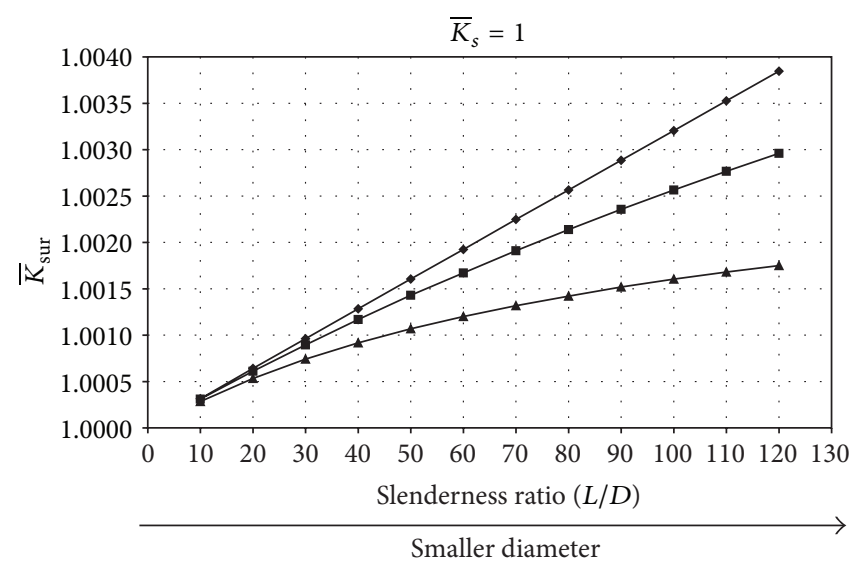

(a)

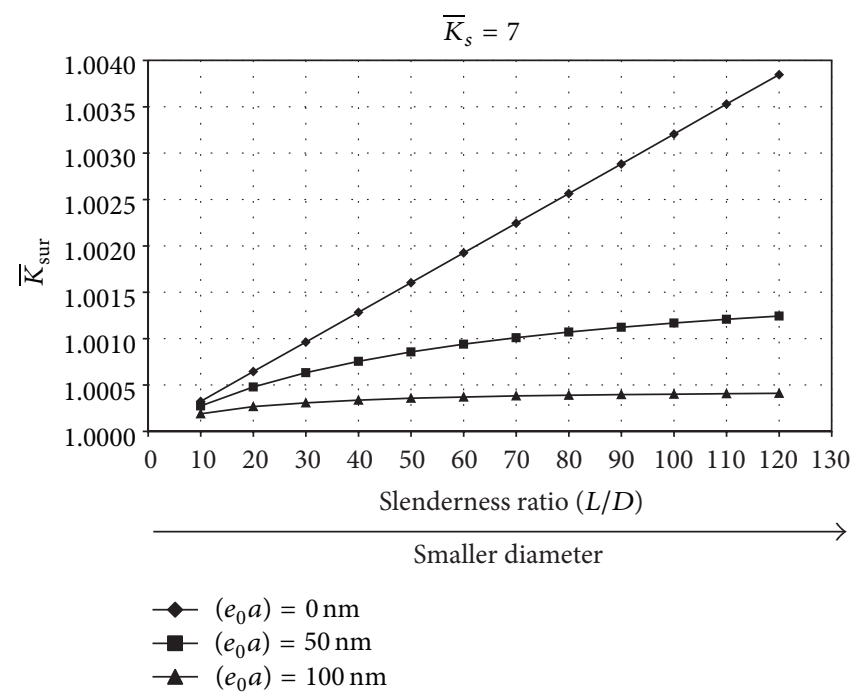

(c)

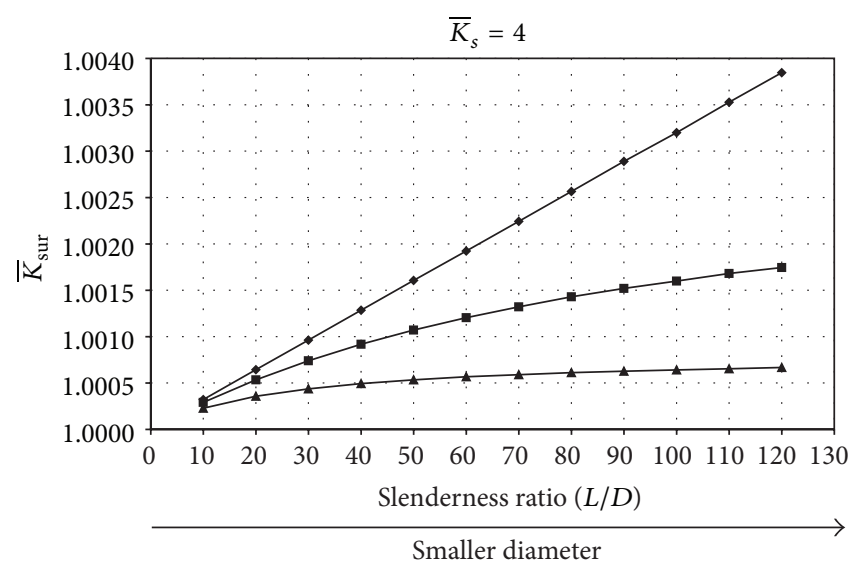

(b)

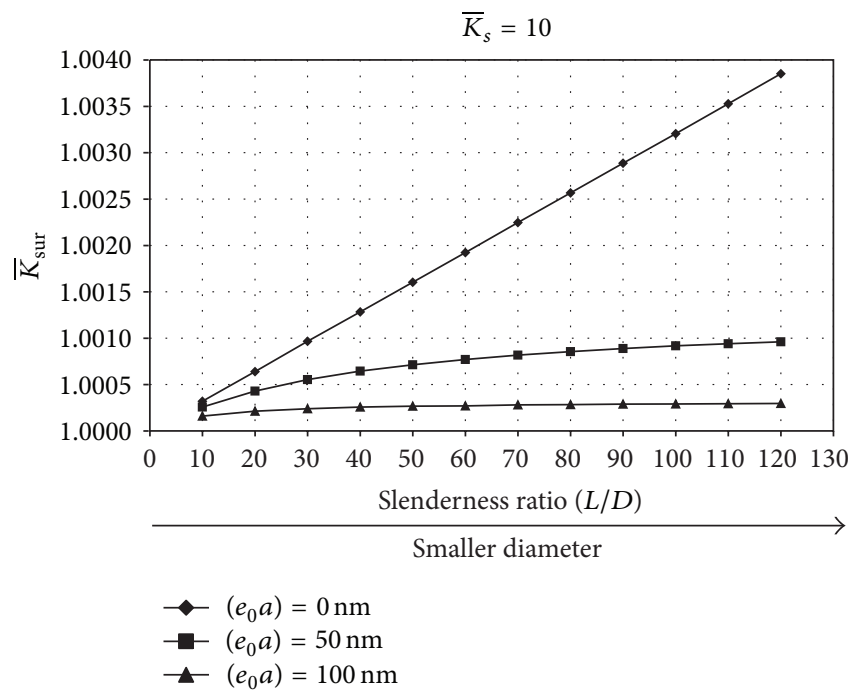

(d)

FIGURE 11: Variation of normalized stiffness $\bar{K}_{\text {sur }}$ with $L / D$ for various elastic substrate stiffness and nonlocal scale parameter.

The equivalent load vector due to nonlocal effect may be written as

$$
\mathbf{P}^{\mathrm{NL}}=\left\{\begin{array}{ll}
P_{1}^{\mathrm{NL}} & P_{2}^{\mathrm{NL}}
\end{array}\right\}^{T}
$$

where

$$
P_{1}^{p_{x}}=-P_{2}^{p_{x}}=\frac{\left(p_{0}-p_{L}\right)}{L}\left(e_{0} a\right)^{2}
$$

The equivalent load vector due to residual surface stress may be written as

$$
\mathbf{P}^{s 0}=\left\{\begin{array}{ll}
P_{1}^{s 0} & P_{2}^{s 0}
\end{array}\right\}^{T}
$$

where

$$
P_{1}^{s 0}=-P_{2}^{s 0}=-\sigma_{x x}^{s 0} \Gamma
$$

\section{Displacement Shape Functions, Axial-Strain-Displacement Shape Functions, and Substrate Deformation-Displacement Shape Functions}

The displacement shape functions may be written as

$$
\begin{gathered}
N_{B 1}(x)=\frac{\sinh [(L-x) \lambda]}{\sinh [\lambda L]}, \\
N_{B 2}(x)=\frac{\sinh [\lambda x]}{\sinh [\lambda L]} .
\end{gathered}
$$

The axial-strain-displacement shape functions may be written as

$$
\begin{gathered}
B_{B 1}(x)=-\frac{\lambda \cosh [(L-x) \lambda]}{\sinh [\lambda L]}, \\
B_{B 2}(x)=\frac{\lambda \cosh [\lambda x]}{\sinh [\lambda L]} .
\end{gathered}
$$


The substrate deformation-displacement shape functions may be written as:

$$
B_{s 1}(x)=N_{B 1}(x), \quad B_{s 2}(x)=N_{B 2}(x) .
$$

\section{Conflict of Interests}

The authors declare that there is no conflict of interests regarding the publication of this paper.

\section{Acknowledgments}

This study was partially supported by the Thailand Research Fund (TRF) under Grant MRG4680109 and Grant RSA5480001 and by the STREAM Research Group under Grant ENG-51-2-7-11-022-S, Faculty of Engineering, Prince of Songkla University. Any opinions expressed in this paper are those of the authors and do not reflect the views of the sponsoring agencies. Special thanks go to senior lecturer Mr. Wiwat Sutiwipakorn for reviewing and correcting the English of this paper.

\section{References}

[1] S. Iijima, "Helical microtubules of graphitic carbon," Nature, vol. 354, no. 6348, pp. 56-58, 1991.

[2] B. Bhushan, Springer Handbook of Nanotechnology, Springer, New York, NY, USA, 3rd edition, 2010.

[3] B. I. Yakobson, C. J. Brabec, and J. Bernholc, "Nanomechanics of carbon tubes: instabilities beyond linear response," Physical Review Letters, vol. 76, no. 14, pp. 2511-2514, 1996.

[4] C. Z. Wang and K. M. Ho, "Tight-binding molecular dynamics for materials simulations," Journal of Computer-Aided Materials Design, vol. 3, no. 1-3, pp. 139-148, 1996.

[5] L. J. D. Frink, A. G. Salinger, M. P. Sears, J. D. Weinhold, and A. L. Frischknecht, "Numerical challenges in the application of density functional theory to biology and nanotechnology," Journal of Physics Condensed Matter, vol. 14, no. 46, pp. 1216712187, 2002.

[6] Q. Wang and V. K. Varadan, "Stability analysis of carbon nanotubes via continuum models," Smart Materials and Structures, vol. 14, no. 1, pp. 281-286, 2005.

[7] A. C. Eringen, "Nonlocal polar elastic continua," International Journal of Engineering Science, vol. 10, no. 1, pp. 1-16, 1972.

[8] A. C. Eringen, "On differential equations of nonlocal elasticity and solutions of screw dislocation and surface waves," Journal of Applied Physics, vol. 54, no. 9, pp. 4703-4710, 1983.

[9] A. C. Eringen, Nonlocal Continuum Field Theories, Springer, New York, NY, USA, 2002.

[10] A. C. Eringen and D. G. B. Edelen, "On nonlocal elasticity," International Journal of Engineering Science, vol. 10, no. 3, pp. 233-248, 1972.

[11] J. Peddieson, G. R. Buchanan, and R. P. McNitt, "Application of nonlocal continuum models to nanotechnology," International Journal of Engineering Science, vol. 41, no. 3-5, pp. 305-312, 2003.

[12] C. M. Wang, Y. Y. Zhang, S. S. Ramesh, and S. Kitipornchai, "Buckling analysis of micro- and nano-rods/tubes based on nonlocal Timoshenko beam theory," Journal of Physics D, vol. 39, no. 17, article 029, pp. 3904-3909, 2006.
[13] J. N. Reddy, "Nonlocal theories for bending, buckling and vibration of beams," International Journal of Engineering Science, vol. 45, no. 2-8, pp. 288-307, 2007.

[14] P. Lu, H. P. Lee, C. Lu, and P. Q. Zhang, "Application of nonlocal beam models for carbon nanotubes," International Journal of Solids and Structures, vol. 44, no. 16, pp. 5289-5300, 2007.

[15] C. Juntarasaid, T. Pulngern, and S. Chucheepsakul, "Bending and buckling of nanowires including the effects of surface stress and nonlocal elasticity," Physica E, vol. 46, pp. 69-76, 2012.

[16] Y. Yang and C. W. Lim, "Non-classical stiffness strengthening size effects for free vibration of a nonlocal nanostructure," International Journal of Mechanical Sciences, vol. 54, no. 1, pp. 57-68, 2012.

[17] P. Malekzadeh and M. Shojaee, "Surface and nonlocal effects on the nonlinear free vibration of non-uniform nanobeams," Composites B, vol. 52, pp. 84-92, 2013.

[18] A. Tounsi, S. Benguediab, E. A. A. Bedia, A. Semmah, and M. Zidour, "Nonlocal effects on thermal buckling properties of double-walled carbon nanotubes," Advances in Nano Research, vol. 1, no. 1, pp. 1-11, 2013.

[19] M. E. Gurtin and A. Ian Murdoch, "A continuum theory of elastic material surfaces," Archive for Rational Mechanics and Analysis, vol. 57, no. 4, pp. 291-323, 1975.

[20] M. E. Gurtin and A. Ian Murdoch, "Surface stress in solids," International Journal of Solids and Structures, vol. 14, no. 6, pp. 431-440, 1978.

[21] P. Lu, L. H. He, H. P. Lee, and C. Lu, "Thin plate theory including surface effects," International Journal of Solids and Structures, vol. 43, no. 16, pp. 4631-4647, 2006.

[22] J. He and C. M. Lilley, "Surface effect on the elastic behavior of static bending nanowires," Nano Letters, vol. 8, no. 7, pp. 17981802, 2008.

[23] Y. Fu, J. Zhang, and Y. Jiang, "Influences of the surface energies on the nonlinear static and dynamic behaviors of nanobeams," Physica E, vol. 42, no. 9, pp. 2268-2273, 2010.

[24] J. L. Liu, Y. Mei, R. Xia, and W. L. Zhu, "Large displacement of a static bending nanowire with surface effects," Physica E, vol. 44, no. 10, pp. 2050-2055, 2012.

[25] Y. Cui, Z. Zhong, D. Wang, W. U. Wang, and C. M. Lieber, "High performance silicon nanowire field effect transistors," Nano Letters, vol. 3, no. 2, pp. 149-152, 2003.

[26] Z. L. Wang and J. Song, "Piezoelectric nanogenerators based on zinc oxide nanowire arrays," Science, vol. 312, no. 5771, pp. 243246, 2006.

[27] X. L. Feng, R. He, P. Yang, and M. L. Roukes, "Very high frequency silicon nanowire electromechanical resonators," Nano Letters, vol. 7, no. 7, pp. 1953-1959, 2007.

[28] L. Y. Jiang and Z. Yan, "Timoshenko beam model for static bending of nanowires with surface effects," Physica E, vol. 42, no. 9, pp. 2274-2279, 2010.

[29] Y. Zhang and Y.-P. Zhao, "Adhesive contact of nanowire in threepoint bending test," Journal of Adhesion Science and Technology, vol. 25, no. 10, pp. 1107-1129, 2011.

[30] A. Khajeansari, G. H. Baradaran, and J. Yvonnet, "An explicit solution for bending of nanowires lying on Winkler-Pasternak elastic substrate medium based on the Euler-Bernoulli beam theory," International Journal of Engineering Science, vol. 52, pp. 115-128, 2012.

[31] G.-F. Wang and X.-Q. Feng, "Surface effects on buckling of nanowires under uniaxial compression," Applied Physics Letters, vol. 94, no. 14, Article ID 141913, 2009. 
[32] J. Xiao, S. Y. Ryu, Y. Huang, K.-C. Hwang, U. Paik, and J. A. Rogers, "Mechanics of nanowire/nanotube in-surface buckling on elastomeric substrates," Nanotechnology, vol. 21, no. 8, Article ID 085708, 2010.

[33] Y. Wang, J. Song, and J. Xiao, "Surface effects on in-plane buckling of nanowires on elastometric substrates," Journal of Physics D, vol. 46, no. 12, Article ID 125309, 2013.

[34] H. G. Poulos and E. H. Davis, Pile Foundation Analysis and Design, John Wiley \& Sons, New York, NY, USA, 1980.

[35] S. Limkatanyu, K. Kuntiyawichai, E. Spacone, and M. Kwon, "Nonlinear Winkler-based beam element with improved displacement shape functions," KSCE Journal of Civil Engineering, vol. 17, no. 1, pp. 192-201, 2013.

[36] R. C. Cammarata, "Surface and interface stress effects in thin films," Progress in Surface Science, vol. 46, no. 1, pp. 1-38, 1994.

[37] B. M. Grafov, "Shuttleworth equation for a finite surface strain," Journal of Electroanalytical Chemistry, vol. 471, no. 2, pp. 105108, 1999.

[38] J. O. Smith and O. M. Sidebottom, Elementary Mechanics of Deformable Bodies, Macmillan Company, New York, NY, USA, 1969.

[39] E. Winkler, Die Lehre Von Der Und Festigkeit, Dominicus, Prag, Czech Republic, 1867.

[40] E. Kreyszig, Advanced Engineering Mathematics, John Wiley \& Sons, New York, NY, USA, 7th edition, 1993.

[41] S. Wolfram, Mathematica Reference Guide, Addison-Wesley Publishing Company, Redwood City, California, 1992.

[42] V. B. Shenoy, "Atomistic calculations of elastic properties of metallic fcc ctrytal surfaces," Physical Review B, vol. 74, no. 14, Article ID 149901, 2006.

[43] K. M. Liew, X. Q. He, and S. Kitipornchai, "Predicting nanovibration of multi-layered graphene sheets embedded in an elastic matrix," Acta Materialia, vol. 54, no. 16, pp. 4229-4236, 2006. 

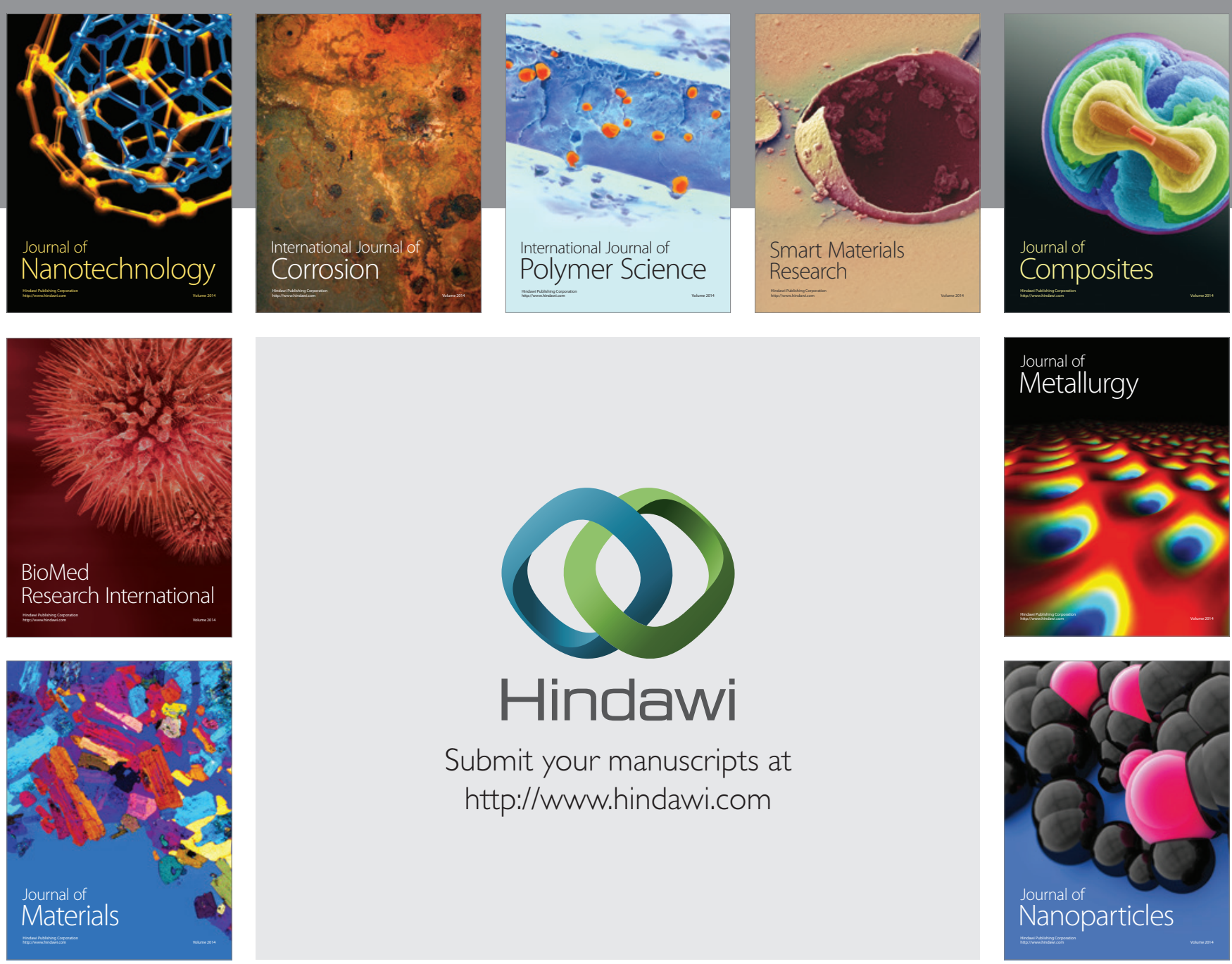

Submit your manuscripts at http://www.hindawi.com
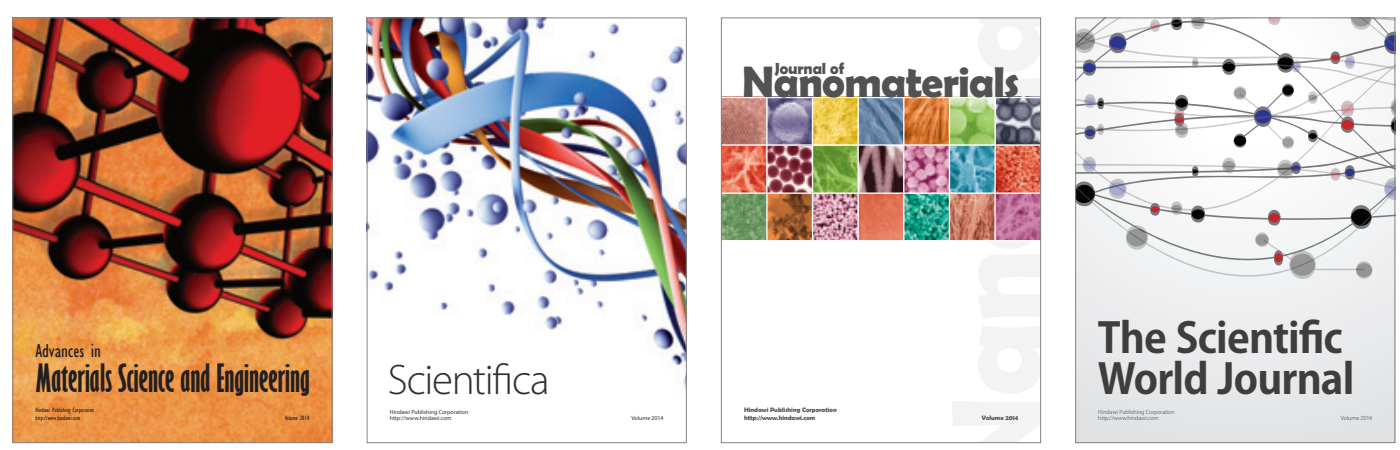

\section{The Scientific World Journal}
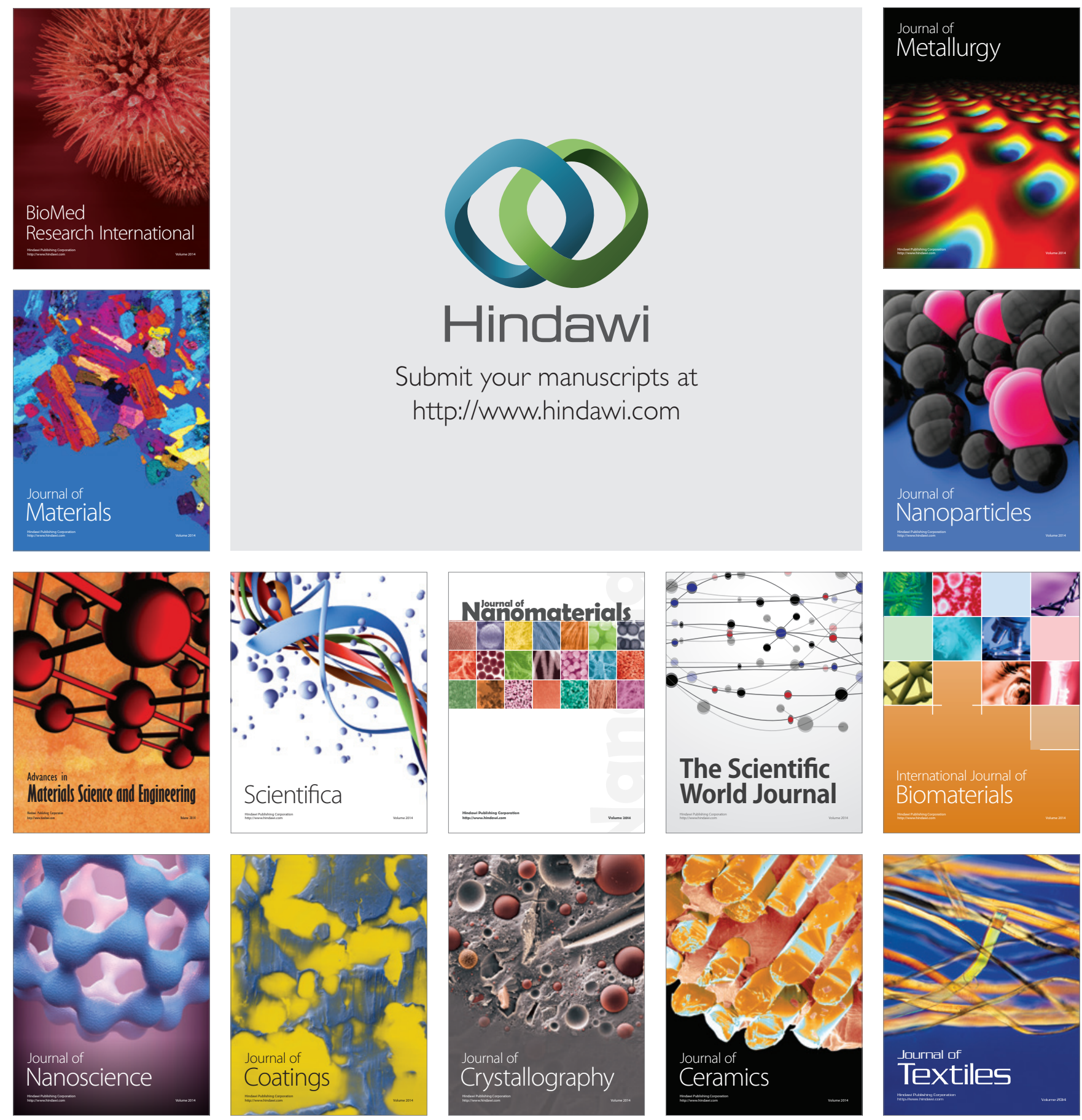